\title{
Spin-noise spectroscopy of a noise-squeezed atomic state
}

\author{
V. Guarrera $\odot,{ }^{1, *}$ R. Gartman $\odot,{ }^{2}$ G. Bevilacqua $\odot,{ }^{3}$ and W. Chalupczak $\odot^{2}$ \\ ${ }^{1}$ Midlands Ultracold Atom Research Centre, School of Physics and Astronomy, University of Birmingham, \\ Edgbaston, Birmingham B15 2TT, United Kingdom \\ ${ }^{2}$ National Physical Laboratory, Hampton Road, Teddington TW11 OLW, United Kingdom \\ ${ }^{3}$ DIISM, Università di Siena, via Roma 56, 53100 Siena, Italy
}

(Received 10 December 2020; accepted 16 June 2021; published 12 July 2021)

\begin{abstract}
Spin-noise spectroscopy is emerging as a powerful technique for studying the dynamics of various spin systems also beyond their thermal equilibrium and linear response. In this context, we demonstrate a nonstandard mode of the spin-noise analysis applied to an out-of-equilibrium nonlinear atomic system realized by a Bell-Bloom atomic magnetometer. Driven by an external pump and undergoing a parametric excitation, this system is known to produce noise squeezing. Our measurements not only reveal a strong asymmetry in the noise distribution of the atomic signal quadratures at the magnetic resonance, but also provide insight into the mechanism behind its generation and evolution. In particular, a structure in the spectrum is identified which allows to investigate the main dependencies and the characteristic timescales of the noise process. The results obtained are compatible with parametrically induced noise squeezing. Notably, the noise spectrum provides information on the spin dynamics even in regimes where the macroscopic atomic coherence is lost, effectively enhancing the sensitivity of the measurements. Our Letter promotes spin-noise spectroscopy as a versatile technique for the study of noise squeezing in a wide range of spin-based magnetic sensors.
\end{abstract}

DOI: 10.1103/PhysRevResearch.3.L032015

Introduction. In standard spin-noise spectroscopy (SNS), spontaneous fluctuations of the atomic spins in thermodynamic equilibrium can provide information on the system properties, such as resonance frequencies and rates of the decoherence processes [1-8]. The extraction of information from the noise in the system is usually based on the fluctuation-dissipation theorem, which relates the spontaneous spin-fluctuation spectrum to the linear response of the atomic system to perturbations [9]. Thus, standard noise studies are specifically performed in unperturbed systems with noise level readout performed via Faraday-rotation type measurements, enabling nondemolition observation of the spin dynamics [10]. In recent years, first attempts to explore SNS beyond the regimes of thermodynamic equilibrium [11-13] and linear response have appeared [14-16]. This is of interest as, for example, standard spin-noise spectra cannot typically disclose information on the system's response to resonant driving fields (out-of-equilibrium dynamics) or reveal the (linear and nonlinear) couplings between the spin coherences associated with the system's relevant energy levels [14].

In the present Letter, we employ SNS in a nonstandard mode to experimentally investigate a nonlinear system driven

\footnotetext{
*v.guarrera@bham.ac.uk

Published by the American Physical Society under the terms of the Creative Commons Attribution 4.0 International license. Further distribution of this work must maintain attribution to the author(s) and the published article's title, journal citation, and DOI.
}

out of equilibrium. We show that the technique can identify the main dependencies and timescales of the noise process, which provides useful information on the system dynamics. More specifically, we test this nonconventional SNS on an atomic spin ensemble exposed to a Bell-Bloom type excitation $[17,18]$. This system undergoes pumping, via optical excitation, and spin-exchange collisions, and, thus, an ensemble polarization is created which precesses around an external magnetic field. In this system, we have previously demonstrated [19] that signal amplification at the Larmor frequency $\omega_{L}$ and noise squeezing are generated at parametric excitation resonances, i.e., for modulation frequencies of the pump beam amplitude which are submultiples of $2 \omega_{L}$. In the model we discussed in Ref. [19], the parametric term enters the individual atoms' spin evolution via a sufficiently strong pump, and its macroscopic effect relies on the synchronized atomic response [20]. With the support of the model we could identify three different regimes of the pump power: a weak pump (standard Bell-Bloom), an intermediate pump (parametric excitation), and a strong pump limit (where few effects, such as a net population transfer between the ground states, excess power broadening, and nonlinear effects due to the pump could impact on the parametric effect). Also, we recall that in the case of modulation frequency of the driving pump $\omega_{M} \neq \omega_{L} / n$, the system dynamics can be characterized by two timescales: a transient where the system oscillates at $\omega_{L}$, and which decays due to spin-exchange collisions, and a steady state oscillating at the modulation frequency $\omega_{M}$. According to the model developed, the parametric excitation in proximity of its threshold only appears in the transient dynamics, and it is, thus, a purely out-of-equilibrium effect. On the other hand, 

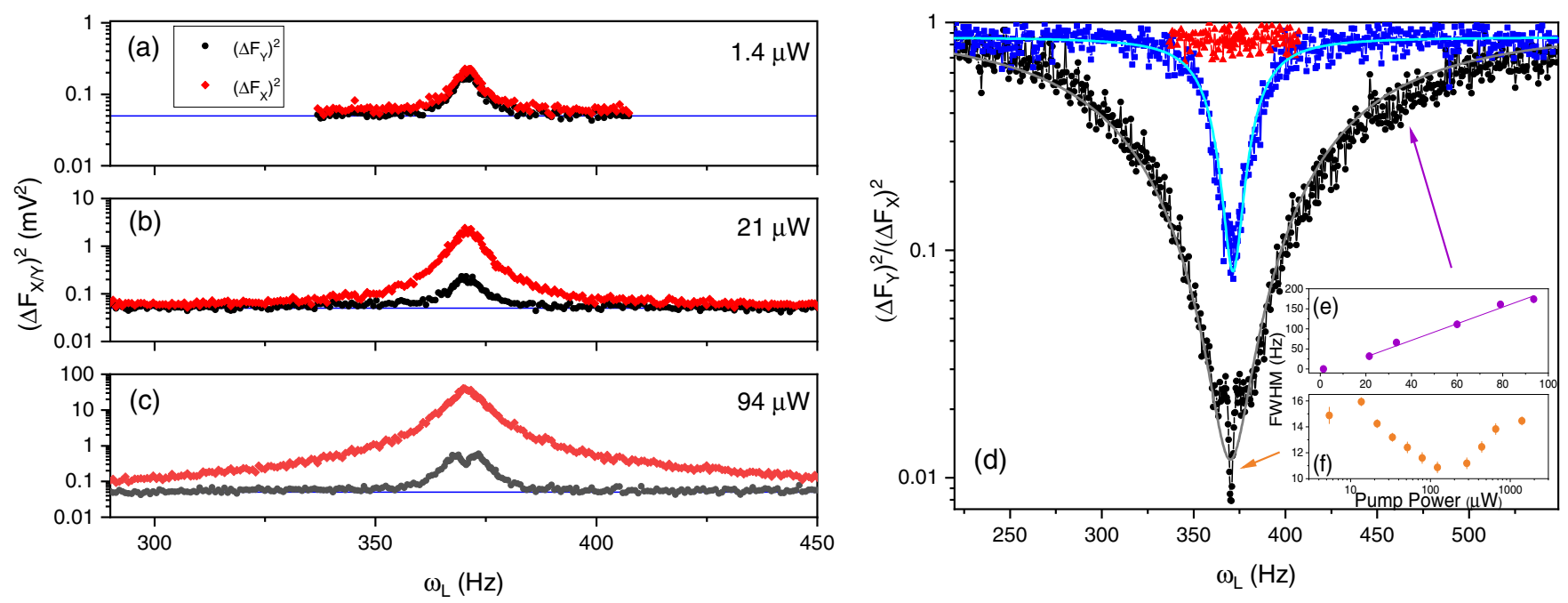

FIG. 1. Variance of the in-phase $\left[\left(\Delta F_{X}\right)^{2}\right.$, red diamonds] and out-of-phase [ $\left(\Delta F_{Y}\right)^{2}$, black dots] amplitudes of the magneto-optical rotation signal oscillating at $\omega$ as a function of the Larmor frequency and recorded for different pump beam powers of (a) $1.4 \mu \mathrm{W}$, (b) $21.5 \mu \mathrm{W}$, and (c) $94 \mu \mathrm{W}$. (d) Dependence of the ratio of the two noise components $\left(\Delta F_{Y}\right)^{2} /\left(\Delta F_{X}\right)^{2}$ on the Larmor frequency recorded with pump beam powers of $1.4 \mu \mathrm{W}$ (red triangles), $21.5 \mu \mathrm{W}$ (blue squares), and $94 \mu \mathrm{W}$ (black dots). Gray/light blue solid lines in (d) are Lorentzian fits to the tails. The insets: (e) full width at half maximum (FWHM) of the broad feature and (f) FWHM of the narrow near-resonance feature as a function of pump power. The signal is acquired with lock-in reference fixed to $\omega=370 \mathrm{~Hz} \approx \omega_{L}$ and time constant of $\tau=100 \mathrm{~ms}$ (e) and $\tau=3 \mathrm{~ms}$ (f), the latter corresponding to the best time resolution of our lock-in amplifier. The optical excitation modulation frequency is equal to $2 \omega$. All the measurements have been performed with probe beam power of $146 \mu \mathrm{W}$. At this power, the probe beam does not have any significant effect on the observed noise spectra.

effects associated with a high pump power might become manifest in the steady-state regime [21].

Here we show that an analysis of the spin noise can confirm these predictions by assisting in the identification of the mechanism which leads to the formation of noise squeezing. As such, on one side our Letter extends the scope of SNS beyond its current boundaries, which has long been appealing from both a theoretical and an experimental point of view $[22,23]$. On the other side, it promotes SNS as a powerful technique for studying spin squeezing. Noise squeezing has attracted much interest in recent years as a strategy to effectively improve sensors' performances beyond those set by classical and standard quantum limits. It has been achieved in several out-of-equilibrium nonlinear systems where, so far, spin fluctuations have mainly been characterized for the quantification of the amount of squeezing achieved (for a review see Ref. [24]).

Experimental system. We detect the amplitude fluctuations in the collective spin of an atomic Cs vapor precessing around a static magnetic field at the Larmor frequency $\omega_{L}$. Measurements are performed on a room-temperature vapor housed in a paraffin coated glass cell with an atomic density of $0.33 \times 10^{11} \mathrm{~cm}^{-3}$. The cell is surrounded by five layers of 2-mm-thick $\mu$ metal shields to reduce static magnetic background. A small static magnetic field is created by a Hemholtz pair of coils (along the $z$ axis, see Ref. [19] for further experimental details). The optical pumping is performed by a circularly polarized laser beam frequency locked to the cesium $6^{2} S_{1 / 2} F=3 \rightarrow 6^{2} P_{3 / 2} F^{\prime}=2$ transition (propagating along the $x$ axis). We modulate the amplitude of the pump laser power with a pulse duty cycle of $7 \%$. The signal produced by the $F=4$ ground-state atomic coherences is read out by a probe beam propagating in a direction orthogonal to the pump beam, whose frequency is blue-detuned by roughly $1 \mathrm{GHz}$ with respect to the $6^{2} S_{1 / 2} F=4 \rightarrow 6^{2} P_{3 / 2} F^{\prime}=5$ transition frequency. The probe light transmitted through the cell (along the $y$ axis) is analyzed by a polarimeter and processed by a lock-in amplifier.

In the following we focus our analysis on the case with modulation frequency of the pump close to $2 \omega_{L}$. This particular parametric resonance has been chosen due to the absence of any direct driving contribution to the coherent signal at $\omega_{L}$, i.e., $\omega_{M} \neq \omega_{L} / n$. Also, the sensitivity of the lock-in amplifier in this case can be set to high level, which enables noise studies with high dynamical range across a wide range of pump beam powers. The signal created in the experiment is acquired for up to $30 \mathrm{~s}$, lock in referenced to $\omega=\omega_{M} / 2=370 \mathrm{~Hz}$ and recorded in its in-phase/out-of-phase $(X / Y)$ components. This means that the detected signal takes the form $S(t)=$ $F_{X}(t) \cos (\omega t)+F_{Y}(t) \sin (\omega t)$ in the reference set by the lockin amplifier, where $S(t)$ is proportional to the collective spin component along the probe beam direction. The pump beam amplitude modulation frequency is fixed whereas the strength of the static magnetic field, and, thus, $\omega_{L}$ is scanned across the resonance $\omega=370 \mathrm{~Hz}$. An acquisition board records several hundred of samples of the lock-in outputs and, subsequently, the variances of the $X$ and $Y$ lock-in signal components are calculated at a time $T$ from the starting of the optical pumping. We note that, when performing the SNS measurements well into the steady-state regime (which is also instrumental for 
ideal lock-in resolution), the coherent signal of the collective atomic spin is mainly lost [21] as if the atomic system was effectively unpolarized.

However, the noise spectrum already shows a clear resonant signal for relatively low pump powers with similar values of the $X$ and $Y$ variances, see Fig. 1(a). Note that with our setup, the spectrum is obtained by varying together $\left(\omega_{L}-\omega_{M}\right)$ and $\left(\omega_{L}-\omega\right)=\left(\omega_{L}-\omega_{M} / 2\right)$ as this allows us to cancel the intrinsic sensitivity of the lock-in amplifier to phase noise of the reference signal and to critically improve the accuracy of the noise detection. The obtained spectra allow us to easily single out the atomic contribution from the total noise. Indeed, driving the Larmor frequency away from the lock-in reference allows to access the background noise [marked with a solid blue line in Figs. 1(a)-1(c)] mainly due to photonic shot noise as we have verified by measuring its linear dependence on the probe beam power.

Pump beam power dependence. In the pump beam power range of $1-4 \mu \mathrm{W}$ the variances of two signal components $X$ and $Y$ do not significantly differ Fig. 1(a) as expected in a standard Bell-Bloom magnetometer. For pump powers above $4 \mu \mathrm{W}$, instead, we start observing an asymmetry in the atomic noise distribution with the noise on the $X$ (in-phase) quadrature being significantly greater than the noise on the $Y$ (out-of-phase) quadrature. Figure 1(b), for example, shows the spin-noise spectrum recorded for a pump beam power of $21.5 \mu \mathrm{W}$ where this asymmetry is clearly visible. Furthermore, for higher pump beam powers (i.e., above $50 \mu \mathrm{W}$ with these lock-in amplifier settings), the spin-noise spectrum shape of the $Y$ component changes with the generation of a dip in correspondence to the resonant frequency $\omega_{L}=\omega_{0}=$ $\omega_{M} / 2$, see Fig. 1(c). We point out that the visibility of this dip depends on the time constant and, hence, on the dynamical response of the lock-in amplifier as we will further discuss in the next paragraph. The observation of asymmetry in the on-resonance noise distribution of conjugated spin variables is a signature of noise squeezing [19]. In fact, we have compared these spectra with those acquired for the same pump powers close to a nonparametric frequency, such as $\omega_{L}=\omega_{M} / 4$ (and $\left.\omega=\omega_{M} / 4\right)$. In this case, the $X$ and $Y$ noise components are always similar, and no asymmetry nor the formation of a dip is observed. A quantitative comparison of the noise level for the same pump power has shown squeezing of the $Y$ component with $\omega_{L} \simeq \omega_{M} / 2$. We note, however, that the values of the squeezed variances are always greater than those measured for a truly unpolarized spin state (by a factor of $\geqslant 1.5$ ). Similar off-resonant quadrature spectra have been used for inferring (quantum) noise squeezing in mechanical systems [25,26].

To isolate the source of the noise asymmetry from other possible nonlinear effects in our noise spectra and help discriminating the dependence on $\omega_{M}$ and $\omega$ in Fig. 1(d) we analyze the ratio between the $Y$ and the $X$ variances, $\left(\Delta F_{Y}\right)^{2} /\left(\Delta F_{X}\right)^{2}$ for different pump powers. With increasing pump powers two main identifiable features emerge in the spectra: a broad Lorentzian profile and a narrow near-resonance feature, black points in Fig. 1(d). When the acquisition time is long enough not to affect the spectral lines, their linewidths provide information about the coherence time of the noise process $[1,7,8]$. We, thus, analyze the FWHM of these two features, and we find that the FWHM of the
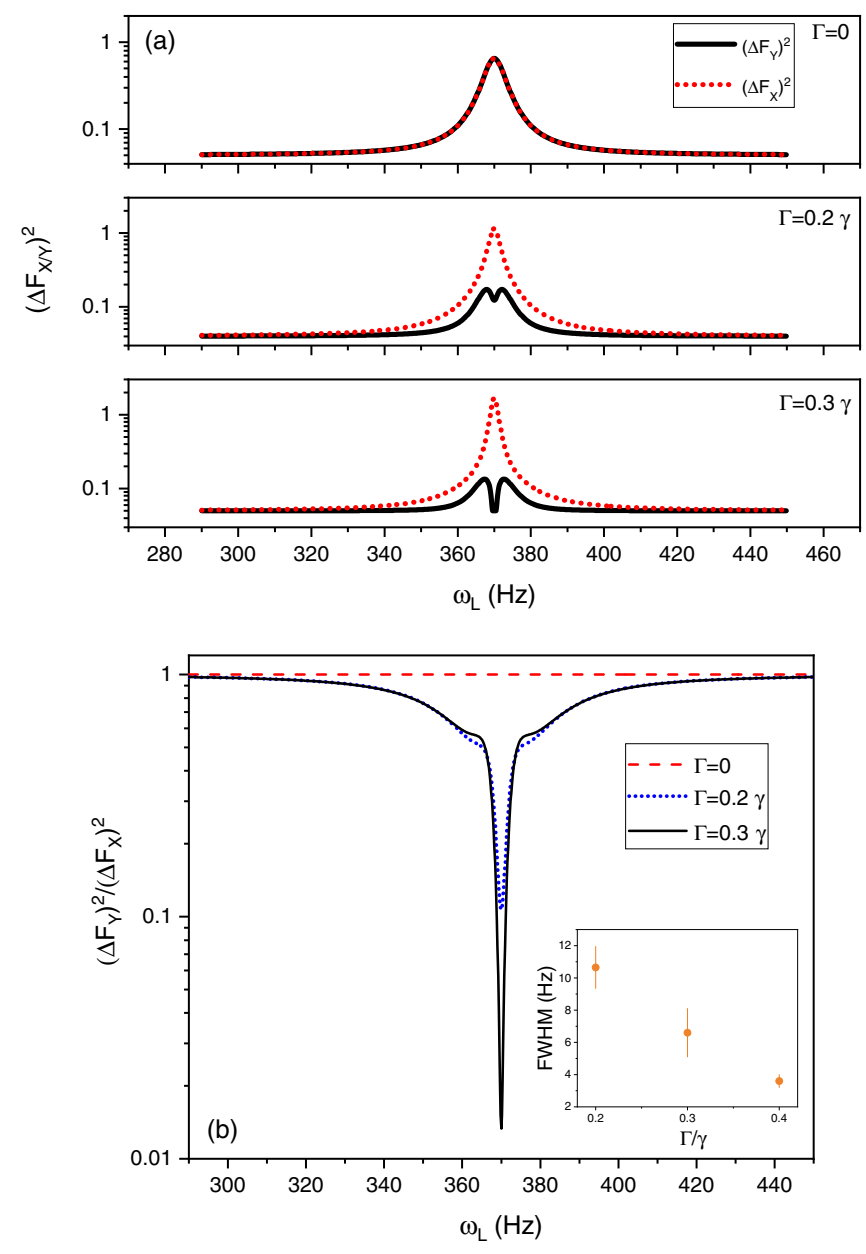

FIG. 2. (a) Parametric model for the in-phase $\left[\left(\Delta F_{X}\right)^{2}\right.$, red dotted line] and out-of-phase $\left[\left(\Delta F_{Y}\right)^{2}\right.$, black solid line] signal variances as a function of the Larmor frequency and calculated for different amplitudes of the parametric term $\Gamma=0,0.2 \gamma, 0.3 \gamma$ with $\gamma$ as the spin coherence relaxation rate due to spin-exchange collisions. (b) Dependence of the ratio of the two noise components $\left(\Delta F_{Y}\right)^{2} /\left(\Delta F_{X}\right)^{2}$ on the Larmor frequency calculated for different values of $\Gamma$. The inset: FWHM of the narrow near-resonance feature as a function of parametric gain. The error bars are obtained from Lorenztian fits of the signals calculated for different $\mathcal{M}$ parameters [28].

broad feature grows with increasing pump power, inset (e) in Fig. 1. In the same range of parameters (for intermediate pump powers $10-100 \mu \mathrm{W}$ ), no such effect of the pump power is detected in the Fourier analysis of the transient oscillation, i.e., varying only $\omega$ [27]. This indicates that the Lorentzian profile mainly contains information about the noise dependence on the excitation frequency around $2 \omega_{L}$. Differently, the FWHM of the near-resonance feature reveals a more subtle dependence on the pump power, Fig. 1(f). In particular, it shows a nonmonotonic behavior, and the FWHM decreases for increasing values of the pump in the range of 10-100 $\mu \mathrm{W}$.

According to the model in Ref. [19], in the system above, power broadening due to the amplitude modulated pump beam is responsible for a parametric contribution to the spin coherence relaxation rate with amplitude $\Gamma$ proportional to the beam intensity. In Figs. 2(a) and 2(b), we show the 

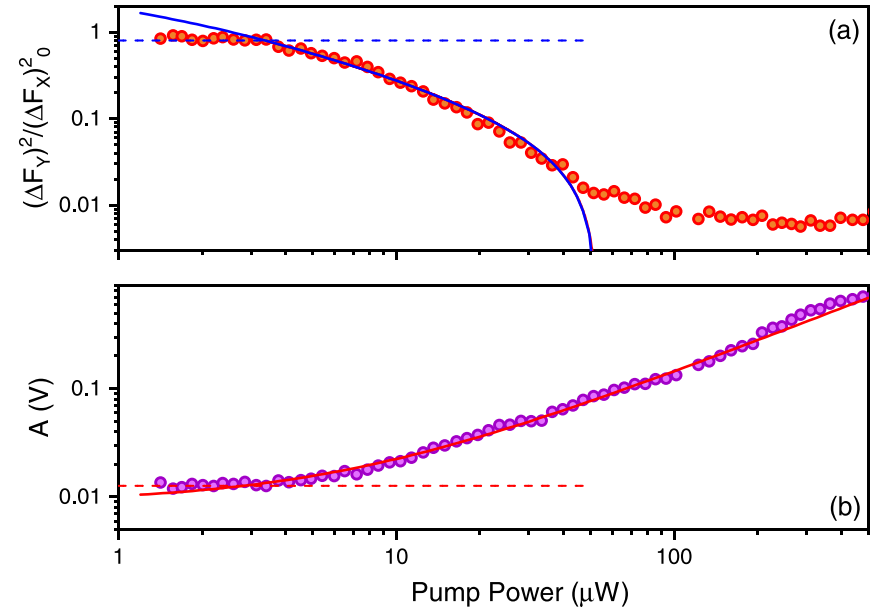

FIG. 3. (a) Variances ratio measured on resonance, i.e., for $\omega_{L}=$ $\omega=\omega_{M} / 2$ as a function of the pump power. The solid line is a fit with the function in Eq. (1), and the dashed line is a fit of the initial plateau. Indication of parametric-induced noise squeezing is observed for pump power below $\omega=370 \mathrm{~Hz}$, in agreement with Ref. [19]. (b) Integrated noise signal as a function of the pump beam power. The solid line is a fit with a linear function, and the dashed line is a fit of the initial plateau.

results obtained with a simple analytical model for a parametric spin system with sinusoidal modulation of the relaxation rate. Good qualitative agreement is found with the experimental data close to resonance in the steady-state regime. Even though the simple model is not expected to properly describe the tails of the spectra where the dependence on $\omega_{M}$ plays a major role, we note that the measured functional dependence of the FWHM of the broad feature is compatible with the linear scaling of the excitation window with the amplitude of the parametric term expected for a detuned parametric oscillator $[29,30]$.

Using this model for interpreting the near-resonance feature, we are allowed to draw some conclusion about the lifetime of the noise-squeezing process, which increases as a function of the strength of the parametric term [Fig. 1(d) and the inset of Fig. 2(b)]. This is clearly different from what is expected from standard power broadening due to the pump. In addition, we note that for higher pump powers, the linewidth of the near-resonance feature starts increasing again, which is not caught by the model and might signal the onset of a different regime where the lifetime of the noise-squeezing process is progressively reduced, leading to a saturation of the observed noise asymmetry. Indeed, a more quantitative analysis of the noise asymmetry on resonance, see Fig. 3(a), shows a threshold behavior around $4 \mu \mathrm{W}$ and a saturation at about $60 \mu \mathrm{W}$, corresponding to a maximum squeezing factor of 13 in variance. For the case of a parametric amplification via modulation of the relaxation rate of the oscillator, we expect (see also Ref. [31])

$$
\frac{\left(\Delta F_{Y}\right)^{2}}{\left(\Delta F_{X}\right)^{2}} \propto \frac{1-g}{1+g}
$$

with $g=\Gamma / \gamma$ the parametric gain. A fit with Eq. (1) provides good agreement with our data in the range of $4-50 \mu \mathrm{W}$.
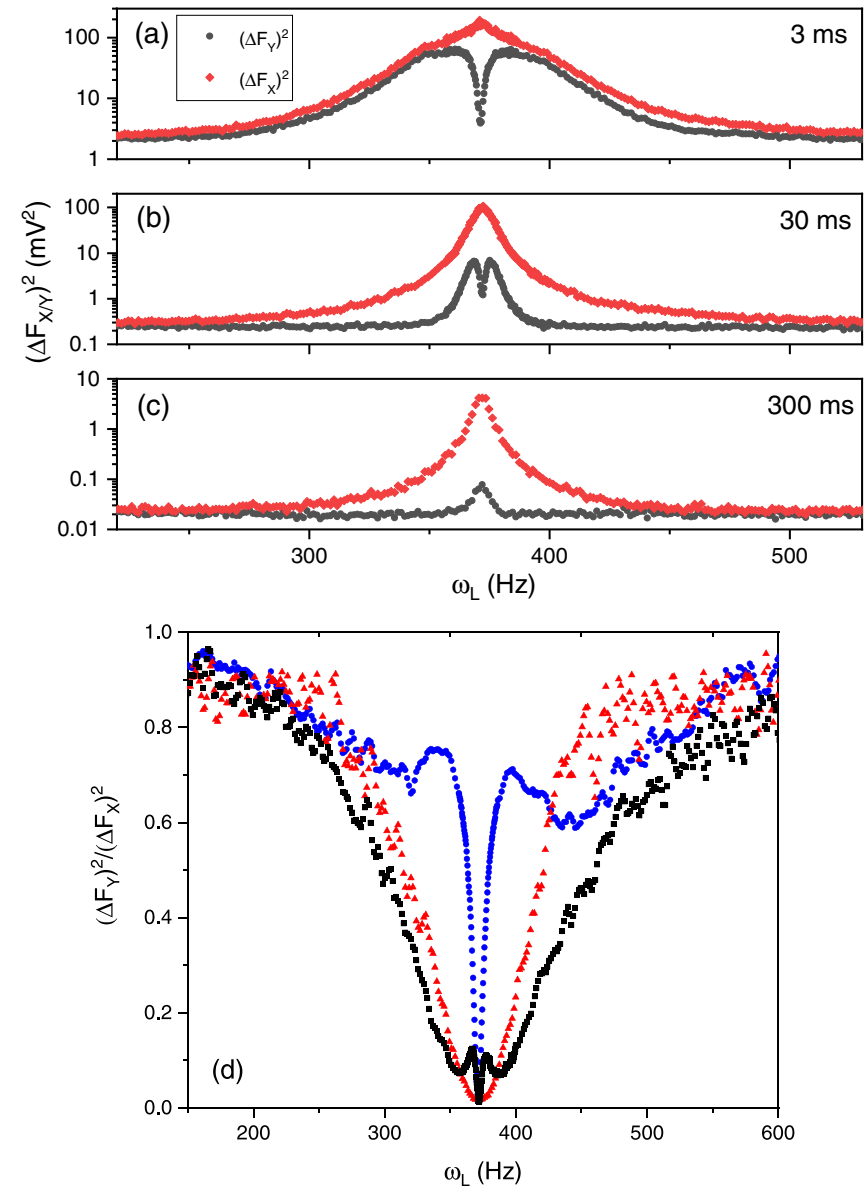

FIG. 4. Dependence of the signal variance on Larmor frequency recorded with the lock-in time constants (a) 3, (b) 30, and (c) 300 ms (lock in referenced to $\omega=370 \mathrm{~Hz}$ ). (d) Dependence of the ratio of the two noise quadratures $\left(\Delta F_{Y}\right)^{2} /\left(\Delta F_{X}\right)^{2}$ on the lock-in time constant with $\tau=3 \mathrm{~ms}$ (blue dots), $30 \mathrm{~ms}$ (black squares), and 300 ms (red triangles). The measurements were made with pump power of $79 \mu \mathrm{W}$ and probe power of $146-\mu \mathrm{W}$ beam power.

Finally, even though in the timescale of these measurements, the average signal $\left\langle F_{X}(t)\right\rangle=\left\langle F_{Y}(t)\right\rangle \approx 0$, we can deduce information about the system susceptibility to develop coherences oscillating at $\omega \simeq \omega_{L}$ from the integrated noise, $A=\int(\Delta F)^{2} d \omega[1]$. We observe that $A$ grows linearly with the pump power above roughly $\sim 4 \mu \mathrm{W}$, see Fig. 3(b). This indicates that the noise asymmetry does not grow unbounded with the coherences generated by the pump.

Temporal dependence. In Fig. 4 we show the noise spectra recorded with three lock-in time constants (a) $\tau=3 \mathrm{~ms}$, (b) $\tau=30 \mathrm{~ms}$, and (c) $\tau=300 \mathrm{~ms}$, leaving the other parameters unvaried (i.e., pump, probe beam powers, and atomic density). The use of relatively low time constants, such as $3 \mathrm{~ms}$, reduces the amount of time averaging performed by the lock-in amplifier and provides similar values of the $X-Y$ variances outside the magnetic resonance, see Fig. 4(a). Synchronization of the signal phase with the modulation frequency of the optical excitation and lock-in reference takes place in the vicinity of the resonance where the narrow structure appears in the spin-noise spectra [Figs. 4(a) and 4(b)]. For a long lock-in time constant we observe strong out-of-resonance averaging 
(a)
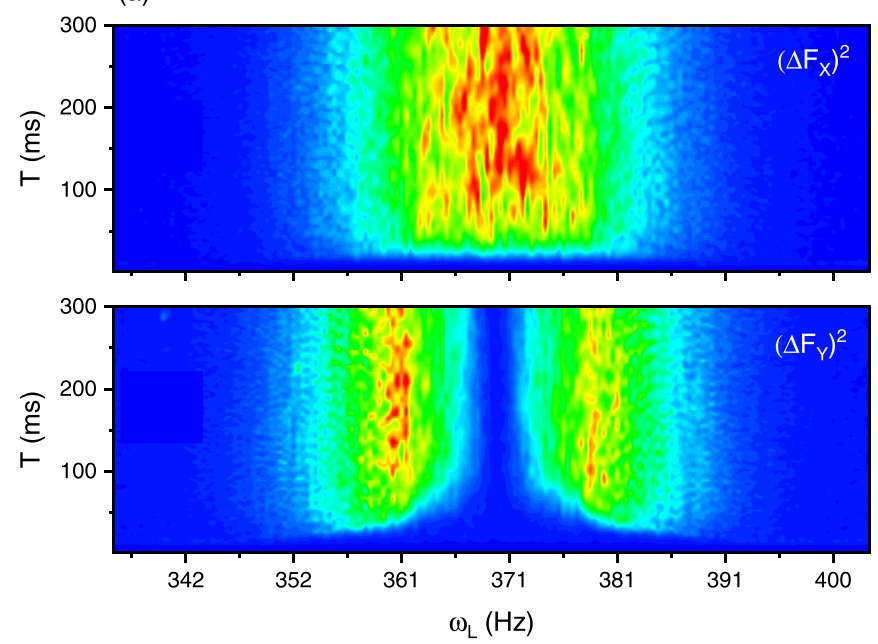

(b)

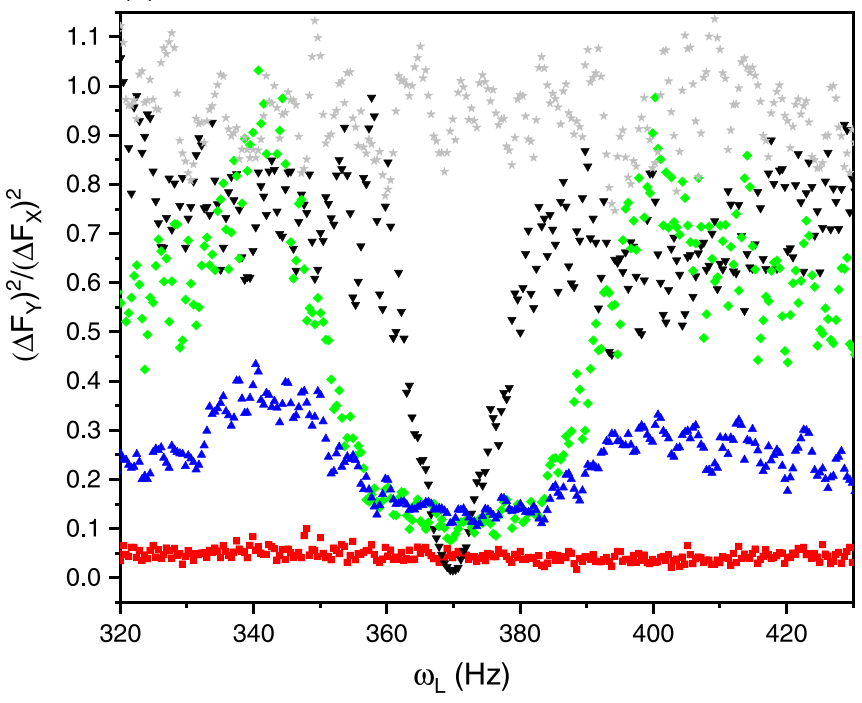

FIG. 5. (a) Dependence of the noise spectra on the total acquisition time, recorded with lock-in time constant of $3 \mathrm{~ms}$ (lock in referenced to $\omega=370 \mathrm{~Hz}$ ) and pump power of $79 \mu \mathrm{W}$. Note that the color scales are different for the two quadratures. (b) Spectra of the two noise components' ratio $\left(\Delta F_{Y}\right)^{2} /\left(\Delta F_{X}\right)^{2}$ for different acquisition times, starting from the beginning of the pumping process: $1 \mathrm{~ms}$ (gray stars), $5 \mathrm{~ms}$ (red squares), $10 \mathrm{~ms}$ (blue triangles), $15 \mathrm{~ms}$ (green diamonds), and $100 \mathrm{~ms}$ (black upside-down triangle).

of the noise fluctuations in the spectrum, Fig. 4(c), whereas the spectrum immediately around the resonance appears more robust. An analysis of the ratio $\left(\Delta F_{Y}\right)^{2} /\left(\Delta F_{X}\right)^{2}$ shows a different time dependence of the two main components. In particular, the Lorentzian profile emerges from longer time averaging as a narrower band of the atomic noise is filtered by the lock-in amplifier. Instead, the near-resonance feature becomes progressively less visible as increasing the lock-in time constant corresponds to binning the signal on a timescale comparable $(\tau=30 \mathrm{~ms})$ or larger $(\tau=300 \mathrm{~ms})$ than the lifetime of the noise process.

To get further insight into the dynamics of the noise generation, we analyze the evolution of the noise spectra from the starting of the pumping process Fig. 5(a). The measured noise for the two quadratures mainly varies within the first $\sim 100 \mathrm{~ms}$ and then does not change up to our maximum observation time $T$ of several seconds. We note that in these measurements the amplitude of the signal at $\omega_{L}$ decays on a comparable timescale of $T_{2}=(94 \pm 1) \mathrm{ms}$, which mainly depends on the rate of the spin-exchange collisions. The ratio between the two quadrature variances reveals an asymmetric noise distribution already after a few milliseconds, Fig. 5(b). The near-resonance feature grows for longer observation times, whereas its FWHM decreases, up to roughly $T=100 \mathrm{~ms}$. This shows that the process generating the asymmetric noise distribution, and, hence, the noise squeezing is limited by the acquisition time $T$ up to roughly $100 \mathrm{~ms}$ which is compatible with the spectrally derived lifetime of $1 / \mathrm{FWHM}=(86 \pm$ 2) $\mathrm{ms}$ and the dependencies on the lock-in time constant, discussed above. This shows that the noise process leading to squeezing is limited to the system transient dynamics as implied by the parametric model of Ref. [19]. As seen above, the saturation of the squeezing is related to a reduction of the lifetime of the noise process, which suggests that decoherence processes, such as those due to excess power broadening (with broadening of the directly pumped coherences $\gtrsim 1 / T_{2}$ ), and nonlinear effects of the pump might play a role.

Conclusions. The characterization of the noise spectrum that we have carried out confirms that the observed noise squeezing is due to the parametric excitation generated by the modulation of the pump beam power and it is mainly limited by the damping due to the finite lifetime of the atomic coherences. Thermal atomic vapors create a platform for a wide range of precise atomic sensors with performances often limited by atomic projection noise [32]. Understanding of the processes which contribute to the signal/noise generation and their dynamics assists in improving these devices [33]. The analysis of the noise spectra of out-of-equilibrium atomic ensembles allows not only to quantify the amount of noise squeezing, but it also provides insight into the processes that contribute to the generation of the noise distribution. We have verified this on a parametrically driven system, which extends the current application of SNS and promotes SNS as a desirable tool for the investigation of spin squeezing in nonlinear out-of-equilibrium systems.

Acknowledgments. The work was funded by the UK Department for Business, Energy and Industrial Strategy (BEIS) as part of the UK National Measurement System Programme. V.G. was supported by the Engineering and Physical Sciences Research Council (Grant No. EP/S000992/1). We would like to thank R. Hendricks and G. Barontini for critical reading of the Letter. G.B. thanks V. Biancalana for fruitful discussions.
[1] S. A. Crooker, D. G. Rickel, A. V. Balatsky, and D. L. Smith, Spectroscopy of spontaneous spin noise as a probe of spin dynamics and magnetic resonance, Nature (London) 431, 49 (2004). 
[2] B. Mihaila, S. A. Crooker, D. G. Rickel, K. B. Blagoev, P. B. Littlewood, and D. L. Smith, Quantitative study of spin noise spectroscopy in a classical gas of $41 \mathrm{~K}$ atoms, Phys. Rev. A 74, 043819 (2006).

[3] G. E. Katsoprinakis, A. T. Dellis, and I. K. Kominis, Measurement of transverse spin-relaxation rates in a rubidium vapor by use of spin-noise spectroscopy, Phys. Rev. A 75, 042502 (2007).

[4] W. Chalupczak and R. M. Godun, Near-resonance spin-noise spectroscopy, Phys. Rev. A 83, 032512 (2011).

[5] V. S. Zapasskii, A. Greilich, S. A. Crooker, Yan Li, G. G. Kozlov, D. R. Yakovlev, D. Reuter, A. D. Wieck, and M. Bayer, Optical Spectroscopy of Spin Noise, Phys. Rev. Lett. 110, 176601 (2013).

[6] N. A. Sinitsyn and Y. V. Pershin, The theory of spin noise spectroscopy: A review, Rep. Prog. Phys. 79, 106501 (2016).

[7] E. L. Ivchenko, Fluctuations of spin polarization of free carriers in semiconductors, Sov. Phys. Semicond. 7, 998 (1974).

[8] E. B. Aleksandrov and V. S. Zapasskii, Magnetic resonance in the Faraday-rotation noise spectrum, JETP 54, 64 (1981).

[9] H. B. Callen and T. A. Welton, Irreversibility and generalized noise, Phys. Rev. 83, 34 (1951).

[10] Y. Takahashi, K. Honda, N. Tanaka, K. Toyoda, K. Ishikawa, and T. Yabuzaki, Quantum nondemolition measurement of spin via the paramagnetic Faraday rotation, Phys. Rev. A 60, 4974 (1999).

[11] I. I. Ryzhov, S. V. Poltavtsev, K. V. Kavokin, M. M. Glazov, G. G. Kozlov, M. Vladimirova, D. Scalbert, S. Cronenberger, A. V. Kavokin, A. Lemaître, J. Bloch, and V. S. Zapasskii, Measurements of nuclear spin dynamics by spin-noise spectroscopy, Appl. Phys. Lett. 106, 242405 (2015).

[12] H. Horn, G. M. Muller, E. M. Rasel, L. Santos, J. Hubner, and M. Oestreich, Spin-noise spectroscopy under resonant optical probing conditions: Coherent and nonlinear effects, Phys. Rev. A 84, 043851 (2011).

[13] S. V. Poltavtsev, I. I. Ryzhov, M. M. Glazov, G. G. Kozlov, V. S. Zapasskii, A. V. Kavokin, P. G. Lagoudakis, D. S. Smirnov, and E. L. Ivchenko, Spin noise spectroscopy of a single quantum well microcavity, Phys. Rev. B 89, 081304(R) (2014).

[14] P. Glasenapp, N. A. Sinitsyn, Luyi Yang, D. G. Rickel, D. Roy, A. Greilich, M. Bayer, and S. A. Crooker, Spin Noise Spectroscopy Beyond Thermal Equilibrium and Linear Response, Phys. Rev. Lett. 113, 156601 (2014).

[15] A. V. Poshakinskiy and S. A. Tarasenko, Spin noise at electron paramagnetic resonance, Phys. Rev. B 101, 075403 (2020).

[16] M. Swar, D. Roy, D. Dhanalakshmi, S. Chaudhuri, S. Roy, and H. Ramachandran, Measurements of spin properties of atomic systems in and out of equilibrium via noise spectroscopy, Opt. Express 26, 32168 (2018).

[17] W. E. Bell and A. L. Bloom, Optically Driven Spin Precession, Phys. Rev. Lett. 6, 280 (1961).
[18] R. Gartman and W. Chalupczak, Amplitude-modulated indirect pumping of spin orientation in low-density cesium vapor, Phys. Rev. A 91, 053419 (2015).

[19] V. Guarrera, R. Gartman, G. Bevilacqua, G. Barontini, and W. Chalupczak, Parametric Amplification and Noise Squeezing in Room Temperature Atomic Vapors, Phys. Rev. Lett. 123, 033601 (2019).

[20] T. Fernholz, H. Krauter, K. Jensen, J. F. Sherson, A. S. Sorensen, and E. S. Polzik, Spin Squeezing of Atomic Ensembles via Nuclear-Electronic Spin Entanglement, Phys. Rev. Lett. 101, 073601 (2008)

[21] A small oscillation at $\omega_{L}$ has been recently observed in the steady state due to replenishing of the decaying coherence by off-resonant pumping.

[22] F. Li, Y. V. Pershin, V. A. Slipko, and N. A. Sinitsyn, Nonequilibrium Spin Noise Spectroscopy, Phys. Rev. Lett. 111, 067201 (2013).

[23] M. M. Glazov, M. A. Semina, E. Y. Sherman, and A. V. Kavokin, Spin noise of exciton polaritons in microcavities, Phys. Rev. B 88, 041309(R) (2013).

[24] L. Pezze, A. Smerzi, M. K. Oberthaler, R. Schmied, and P. Treutlein, Quantum metrology with nonclassical states of atomic ensembles, Rev. Mod. Phys. 90, 035005 (2018).

[25] E. E. Wollman, C. U. Lei, A. J. Weinstein, J. Suh, A. Kronwald, F. Marquardt, A. A. Clerk, and K. C. Schwab1, Quantum squeezing of motion in a mechanical resonator, Science $\mathbf{3 4 9}$, 952 (2015)

[26] J. M. Pirkkalainen, E. Damskagg, M. Brandt, F. Massel, and M. A. Sillanpaa, Squeezing of Quantum Noise of Motion in a Micromechanical Resonator, Phys. Rev. Lett. 115, 243601 (2015).

[27] R. Gartman, V. Guarrera, G. Bevilacqua, and W. Chalupczak, Linear and nonlinear coherent coupling in a Bell-Bloom magnetometer, Phys. Rev. A 98, 061401(R) (2018).

[28] See Supplemental Material at https://link.aps.org/supplemental/ 10.1103/PhysRevResearch.3.L032015 for a detailed description of the analytical model for the noise spectrum of a parametric spin system with sinusoidal modulation of the relaxation rate.

[29] L. D. Landau and E. M. Lifshitz, Mechanics (ButterworthHeinemann, Oxford, 1976).

[30] H. J. Carmichael, G. J. Milburn, and D. F. Walls, Squeezing in a detuned parametric amplifier, J. Phys. A 17, 469 (1984).

[31] T. Briant, P. F. Cohadon, M. Pinard, and A. Heidmann, Optical phase-space reconstruction of mirror position at the attometer level, Eur. Phys. J. D 22, 131 (2003).

[32] J. Kitching, S. Knappe, and E. A. Donley, Atomic sensors-A review, IEEE Sens. J. 11, 1749 (2011).

[33] W. Wasilewski, K. Jensen, H. Krauter, J. J. Renema, M. V. Balabas, and E. S. Polzik, Quantum Noise Limited and Entanglement-Assisted Magnetometry, Phys. Rev. Lett. 104, 133601 (2010) 